1885.] Transformation Formula $y=\sin (L+A+B+O+\ldots) . \quad 11$

It is proper to remark that the vibrations here considered are covered by the general theory of spherical vibrations given by Lamb in the paper referred to. But it would probably be as difficult, if not more difficult, to deduce the conclusions of the present paper from the analytical expressions of the general theory, as to obtain them independently. It is not improbable that the surface waves here investigated play an important part in earthquakes, and in the collision of elastic solids. Diverging in two dimensions only, they must acquire at a great distance from the source a continually increasing preponderance.

On some Consequences of the Transformation Formula

$$
y=\sin (L+A+B+O+\ldots) \text {. }
$$

By John Grifriths, M.A.

[Read Nov. 12th, 1885.]

\title{
Contents of Paper.
}

Notatron,-OUthine.

Secr. 1.-On some Developments of the Equation $y=\sin (L+A+B+\ldots)$.

Secr. 2.-Deduction of $\quad y=\frac{\left(1, x^{2}\right)^{n}}{\left(1, x^{2}\right)^{n}}$.

Szcr. 3.-Deduction of $\quad y=\frac{x\left(1, x^{2}\right)^{n-1}}{\left(1, x^{2}\right)^{n}}$.

SBCr. 4.-Deduction of $\quad y=\frac{\left(1, x^{2}\right)^{n}}{x\left(1, x^{2}\right)^{n-1}}$.

Secr. 5.-Complete multiplication by $2 n$. Expression for on $2 n u$ deduced from two conjugate transformations.

Secr. 6.-Imaginary Transformations.

Secr. 7. - Transformation of $\Theta$ and $\Phi$ functions.

Szot. 8.-Complete multiplication by $2 n$. Expression for $\odot 2 n u$.

ApPendix.-Remarks on the modular equation for an even transformation.

The second equation corresponding to an order of transformation $=2 \times$ odd prime number $n$.

\section{Notation.}

In order to aroid repetitions, it is convenient, for the purposes of 
this Note, to write

$$
\begin{gathered}
\cos A=\frac{1-\left(1+a^{3}\right) x^{2}}{1-a^{2} x^{2}}, \quad \sin A=\frac{2 a^{\prime} x \sqrt{1-x^{2}}}{1-a^{3} x^{2}}, \\
\cos B=\frac{1-\left(1+\beta^{\prime 2}\right) x^{2}}{1-\beta^{2} x^{8}}, \& c .,
\end{gathered}
$$

where

$$
a^{2}+a^{\prime 2}=1=\beta^{2}+\beta^{\prime 2}=\ldots .
$$

$$
\begin{gathered}
\sin L=\frac{\left(1+k^{\prime}\right) x \sqrt{1-x^{2}}}{\sqrt{1-k^{2} x^{2}}}, \quad \cos L=\frac{1-\left(1+k^{\prime}\right) x^{2}}{\sqrt{1-k^{2} x^{2}}} . \\
u_{0}=\frac{s K}{n}, \quad v_{0}=\frac{2 t-1}{2 n} K, \quad(\bmod k), \\
u_{0}^{\prime}=\frac{s K^{\prime}}{n}, \quad v_{0}^{\prime}=\frac{2 t-1}{2 n} K^{\prime}, \quad\left(\bmod k^{\prime}\right) ;
\end{gathered}
$$

$s$ being an integer from 1 to $n-1$, and $t$ an integer from 1 to $n$.

$\left(1, x^{2}\right)^{n} \equiv$ rational and integral function of $x$ of the order $2 n$. (Prof. Cayley's notation).

$\Pi \equiv$ product of a certain number of factors ; for example,

$$
\Pi\left\{1-\frac{x^{2}}{\operatorname{sn}^{2} u_{0}}\right\}=\left(1-\frac{x^{2}}{\operatorname{sn}^{2} \frac{K}{n}}\right)\left(1-\frac{x^{2}}{\operatorname{sn}^{2} \frac{2 K}{n}}\right) \cdots \cdots\left(1-\frac{x^{2}}{\operatorname{sn}^{2} \frac{n-1}{n} K}\right)
$$

to $n-1$ factors.

In the case of $n=1, \Pi$ must be taken $\equiv 1$. ct $\equiv \mathrm{cn} \div \mathrm{sn}$.

The object of the Note is, in the first instance, to show how the three even rational transformation equations of the forms

$$
y=\frac{x\left(1, x^{2}\right)^{n-1}}{\left(1, x^{2}\right)^{n}}, \quad y=\frac{\left(1, x^{2}\right)^{n}}{\left(1, x^{2}\right)^{n}}, \quad y=\frac{\left(1, x^{2}\right)^{n}}{x\left(1, x^{2}\right)^{n-1}},
$$

can be derived from the formula

$$
y=\sin (L+A+B+\ldots) ;
$$

and, secondly, to notice some results as regards the transformation and complete multiplication by $2 n$ of the sn and $\theta$ functions. 
1885.] Transformation Formula $y=\sin (L+A+B+O+\ldots) . \quad 13$

SECT. 1.-On some Developments of the Equation

$$
y=\sin (L+A+B+\ldots) \text {. }
$$

Here $y=\frac{x \sqrt{1-x^{2}}}{\sqrt{1-k^{2} x^{2}}} \times$ rational function of $x$, and $\sqrt{1-y^{2}}=$ rational function of $x \div \sqrt{1-k^{2} x^{2}}$, so that Jacobi's change of $y, x$ into $\frac{1}{\lambda y}, \frac{1}{k x}$ is applicable. In fact, the integral equation gives rise to the differential relation

$$
\frac{d y}{\sqrt{1-y^{2} \cdot 1-\lambda^{2} y^{2}}}=M \frac{d x}{\sqrt{1-x^{2} \cdot 1-k^{2} x^{2}}}
$$

provided that

$$
M \sqrt{1-\lambda^{2} y^{2}}=\sqrt{1-k^{2} x^{2}}\left\{1+\frac{k^{\prime}}{1-k^{2} x^{2}}+2 \Sigma \frac{a^{\prime}}{1-a^{2} x^{2}}\right\} .
$$

(See a note by the present writer, Proc. Lond. MFath. Soc., Vol. xV., p. 64.)

But, besides the change of $y, x$ in question, we have, in this instance, the following ones, viz., of

$$
\left.\begin{array}{l}
y \text { into } \lambda y \\
\lambda \text { into } \frac{1}{\lambda} \\
M I \text { into } M \pi \lambda
\end{array}\right\} .
$$

Writing $y=\operatorname{sn}(M u, \lambda)$ and $x=\operatorname{sn}(u, k)$,

we deduce then the groups of formulae,

$$
\begin{aligned}
& y=M x \sqrt{1-x^{2}} \Pi\left\{1-\frac{x^{2}}{\operatorname{sn}^{2} u_{0}}\right\} \div \\
& \sqrt{1-y^{2}}=\Pi\left\{1-\frac{2^{2}}{\operatorname{sn}^{2} v_{0}}\right\} \div \\
& \sqrt{1-\lambda^{2} y^{2}}=\Pi\left\{1-k^{2} \operatorname{sn}^{2} v_{0} x^{2}\right\} \div
\end{aligned}
$$

common denom. $\sqrt{1-k^{2} x^{2}} \Pi\left\{1-k^{2} \operatorname{sn}^{2} u_{0} x^{2}\right\}$

(see notation).

$$
\frac{M \Gamma \lambda}{k^{2}} \cdot \operatorname{sn}(M L, \lambda)=\frac{\operatorname{sn} u \operatorname{cn} u}{\operatorname{dn} u}\left\{1+2 \Sigma \frac{(-)^{\prime} \operatorname{cn}^{2} \frac{s K}{n}}{1-k^{2} \operatorname{sn}^{2} \frac{s K}{n} \operatorname{sn}^{2} u}\right\}
$$




$$
\begin{gathered}
M \lambda . \operatorname{cn}(M u, \lambda)=\operatorname{dn} u\left\{1+\frac{(-)^{n} k^{\prime}}{\operatorname{dn}^{2} u}+2 \Sigma \frac{(-)^{\circ} \mathrm{dn} \frac{s K}{n}}{1-k^{2} \operatorname{sn}^{2} \frac{s K}{n} \operatorname{sn}^{2} u}\right\}, \\
M . \operatorname{dn}(M u, \lambda)=\operatorname{dn} u\left\{1+\frac{k^{\prime}}{\operatorname{dn}^{2} u}+2 \Sigma \frac{\operatorname{dn} \frac{s K}{n}}{1-k^{2} \operatorname{sn}^{2} \frac{s K}{n} \operatorname{sn}^{2} u}\right\} ;
\end{gathered}
$$

where the order of the transformation $=2 n$.

$$
M=\Pi \mathrm{sn}^{2} \frac{s K}{n} \div \Pi \operatorname{sn}^{2} \frac{2 t-1}{2 n} K .
$$

SECT. 2.-Deduction of $Y=\frac{\left(1, x^{2}\right)^{n}}{\left(1, x^{2}\right)^{n}}$ from the above equations.

If we pat $Y=\sqrt{\frac{1-y^{2}}{1-\lambda^{2} y^{2}}}$, we have, from the foregoing Section, a rational transformation equation, of order $2 n$, wherein

$$
\begin{gathered}
Y=\Pi\left\{1-\frac{x^{2}}{\operatorname{sn}^{2} v_{0}}\right\} \div \\
\sqrt{1-Y^{2}}=M \lambda^{\prime} .2 \sqrt{1-x^{2}} \Pi\left\{1-\frac{x^{2}}{\operatorname{sn}^{2} u_{0}}\right\} \div \\
\sqrt{1-\lambda^{2} Y^{2}}=\lambda^{\prime} \sqrt{1-k^{2} x^{2}} \Pi\left\{1-k^{2} \operatorname{sn}^{2} u_{0} x^{2}\right\} \div \\
\text { common denom. }=\Pi\left\{1-k^{2} \operatorname{sn}^{2} v_{0} x^{2}\right\} .
\end{gathered}
$$

Here we have, in fact, a formula

$$
\boldsymbol{Y}=\cos (A+B+O+\ldots)=\frac{\left(1, x^{2}\right)^{n}}{\left(1, x^{2}\right)^{n}}
$$

where the coefficients $\alpha, \beta$, \&c. may be taken to be

$$
\alpha=k \operatorname{sn} \frac{K}{2 n}, \quad a^{\prime}=\operatorname{dn} \frac{K}{2 n}, \quad \beta=k \sin \frac{3 K}{2 n},
$$

and so on for odd multiples of $\frac{K}{2 n}$.

This gives the differential equation

$$
\frac{d Y}{\sqrt{1-Y^{2} \cdot 1-\lambda^{2} Y^{2}}}=-M \frac{d x}{\sqrt{1-x^{2} \cdot 1-k^{2} x^{2}}} .
$$


1885.] Transformation Formula $y=\sin (L+A+B+O+\ldots) . \quad 15$

Hence $\quad \Lambda-\int_{0}^{Y} \frac{d Y}{\sqrt{1-Y^{2} \cdot 1-\lambda^{2} Y^{2}}}=M u$, or, say, $\Lambda-v_{\lambda}=M u$.

If, then, $\int_{0}^{y} \frac{d y}{\sqrt{1-y^{8} \cdot 1-\lambda^{8} y^{2}}}=u_{\lambda}$ and $\int_{0}^{Y} \frac{d Y}{\sqrt{1-Y^{2} \cdot 1-\lambda^{9} Y^{8}}}=v_{\lambda}$,

the relation between the two transformations is

$$
u_{\lambda}+v_{\lambda}=\Lambda, \quad(\bmod \lambda) \text {. }
$$

SECr. 3. Deduction of $z=\frac{x\left(1, x^{8}\right)^{n-1}}{\left(1, x^{2}\right)^{n}}$.

From $y=\sin (L+A+\ldots)$, I have already derived the secondary transformation $i z=\tan \left(X_{0}+X_{1}+\ldots+X_{n-1}\right)$, where $\quad \tan X_{0}=i \frac{(1+k) x}{1+k x^{2}}, \quad \tan X_{1}=\frac{2 i a_{1} x}{1+a_{1}^{2} x^{2}}$, \&c., and the coefficients are of the form

$$
a_{s}=\operatorname{dn}\left(\frac{s K^{\prime}}{n}, k^{\prime}\right) \text {. }
$$

See Proc. Lond. Math. Soc., Vol. xvi., p. 90.

This gives $\int_{0}^{\infty} \frac{d z}{\sqrt{1-z^{2} \cdot 1-\gamma^{8} z^{2}}}=N \int_{0}^{x} \frac{d x}{\sqrt{1-x^{3} \cdot 1-h^{3} x^{2}}}$, or, say, $\quad u_{r}=N u$.

We have, accordingly,

$$
\begin{gathered}
N=\Pi \sin ^{2}\left(\frac{s K^{\prime}}{n}, k^{\prime}\right) \div \Pi \operatorname{sn}^{2}\left(\frac{2 t-1}{2 n} K^{\prime}, k^{\prime}\right) ; \\
\operatorname{sn}(N u, \gamma)=N \operatorname{sn} u . \Pi\left\{1+c t^{2} u_{0}^{\prime} \operatorname{sn}^{2} u\right\} \div \\
\operatorname{cn}(N u, \gamma)=\operatorname{cn} u \operatorname{dn} u . \Pi\left\{1-\operatorname{dn}^{2} u_{0}^{\prime} \operatorname{sn}^{2} u\right\} \div
\end{gathered}
$$

$\operatorname{dn}(N u, \gamma)=\Pi\left\{1-\operatorname{dn}^{3} v_{0}^{\prime} \operatorname{sn}^{2} u\right\} \div$ common denom. $\Pi\left\{1+\operatorname{ct}^{3} v_{0}^{\prime} \operatorname{sn}^{3} u\right\}$. (For the values of $u_{0}^{\prime}$ and $v_{0}^{\prime}$, see notation.)

For example, when $2 n=6$,

$$
\begin{aligned}
\operatorname{sn}(N u, \gamma)= & N \operatorname{sn} u\left(1-k^{2} \operatorname{sn}^{2} \frac{i K^{\prime}}{3} \operatorname{sn}^{2} u\right)\left(1-k^{9} \operatorname{sn}^{2} \frac{2 i K^{\prime}}{3} \sin ^{2} u\right) \\
& +\left(1+k i x^{2}\right)\left(1-k^{2} \sin ^{2} \frac{i K^{\prime}}{6} \sin ^{3} u\right)\left(1-k^{3} \operatorname{sn}^{2} \frac{5 i K^{\prime}}{6} \operatorname{sun}^{2} u\right) .
\end{aligned}
$$


SECr. 4.-Deduction of $Z=\frac{\left(1, x^{2}\right)^{n}}{x\left(1, x^{2}\right)^{n-1}}$.

From Sect. 2 therc follows a secondary equation,

$$
\begin{aligned}
& Z=i \cot \left(X_{1}+X_{2}+\ldots+X_{n}\right), \text { where } \tan X_{1}=\frac{2 i b_{1} v}{1+b_{1}^{2} x^{2}}, \\
& \tan X_{2}=\frac{2 i b_{2} x}{1+b_{2}^{2} 2^{2}}, \& c ., \text { and } b_{t}=\operatorname{dn}\left(\frac{2 t-1}{2 \imath} K^{\prime}, k^{\prime}\right) ;
\end{aligned}
$$

$t$ being an integer from 1 to $n$.

Here $\quad \int_{\infty}^{z} \frac{d Z}{\sqrt{1-Z^{2} \cdot 1-\gamma^{2} Z^{2}}}=N \int_{0}^{x} \frac{d x}{\sqrt{1-x^{2} \cdot 1-k^{2} x^{2}}}$

i.e.,

$$
v_{y}=i \Gamma^{\prime}+N u \text {, }
$$

or

$$
u_{\gamma}+v_{\gamma}=i \Gamma^{\prime}, \quad(\bmod \gamma) . \quad \text { See Sect. } 3 .
$$

Secr. 5.-Complete Multiplication by 2n. Eupression for sn 2 nu deduced from two conjugate Transformations.

Complete multiplication by $2 n$ may be effected in several ways; for instance, writing the equations of Sects. 2 and 4 , as $Y=f(x, k)$, $Z=\phi(\imath, k)$, and taking the modular relations to be

$$
\left.\left.\begin{array}{rlrl}
2 n \Lambda & =N I K \\
\Lambda^{\prime} & =N K^{\prime}
\end{array}\right\} \quad \begin{array}{rl}
\Gamma & =N K \\
2 n \Gamma^{\prime} & =N K^{\prime}
\end{array}\right\},
$$

(see Proc. Lond. Mrath. Soc., Vol. xvi., p. 91,)

$$
\text { we derive } \int_{1}^{Y} \frac{d Y}{\sqrt{1-Y^{2} \cdot 1-\lambda^{2} Y^{2}}}=-M \int_{0}^{x} \frac{d x}{\sqrt{1-x^{2} \cdot 1-k^{2} x^{2}}} \text {; }
$$

change $Y$ into $X ; \curvearrowright$ into $Z ; \lambda, k, \Delta \Gamma$ into $k, \gamma, \frac{2 \pi}{N}$; then

$$
\begin{aligned}
& \int_{1}^{x} \frac{d X}{\sqrt{1-X^{2} \cdot 1-k^{2} X^{2}}}=-\frac{2 n}{N} \int_{0}^{z} \frac{d Z}{\sqrt{1-Z^{2} \cdot 1-\gamma^{2} Z^{3}}}=-\frac{2 n}{N}\left(i \Gamma^{\prime}+N u\right), \\
& \text { i.e., } \quad \int_{0}^{x} \frac{l l X}{\sqrt{ } 1-X^{2} \cdot 1-k^{2} X^{2}}=K-\frac{2 n}{N} i \Gamma^{\prime}-2 n u=K-i K^{\prime}-2 n u \text {, } \\
& \text { or } \quad \sin \left(K-i K^{\prime}-2 n u\right)=f(Z, \gamma) \text {, } \\
& \text { where } \quad Z=\phi(\mathrm{sn} u, h) \text {. }
\end{aligned}
$$


Secr. 6.-Imaginary Transformations.

The general form of the transformations of Sect. 3 appears to be

$$
\begin{aligned}
\operatorname{sn}(N u, \gamma)= & N \text { sn } u . \Pi\left\{1-k^{2} \operatorname{sn}^{2} \frac{2 p K+p^{\prime} i K^{\prime}}{n} \operatorname{sn}^{2} u\right\} \\
& \div \Pi\left\{1-k^{2} \operatorname{sn}^{2} \frac{2 m K+\left(2 m^{\prime}+1\right) i K^{\prime}}{2 n} \operatorname{sn}^{2} u\right\},
\end{aligned}
$$

if $p, p^{\prime}, m, m^{\prime}$ be certain integers, positive and negative, or, say,

$$
\operatorname{sn}(N u, \gamma)=N \sin u\left(1, \operatorname{sn}^{2} u\right)^{n-1} \div\left(1, \operatorname{sn}^{2} u\right)^{n} .
$$

The functions $\left(1, \operatorname{sn}^{2} u\right)^{n-1}$ and $\left(1, \operatorname{sn}^{2} u\right)^{n}$ are self-inverse, i.e., each of them remains unaltered, to a factor près, when $\frac{1}{k \operatorname{sn} u}$ is written therein for sn $u$.

Since it is essential that $\operatorname{cn}(N u, \dot{\gamma})$ shall be $=\operatorname{cn} u \operatorname{dn} u \times$ rational function of $\operatorname{sn} u$, the integers must in all cases satisfy the relation

$$
\Pi \mathrm{dn}^{4} \frac{2 m i K^{\prime}-\left(2 m^{\prime}+1\right) K}{2 n}=\left(k^{\prime}\right)^{2} \Pi \mathrm{dn}^{4} \frac{2 p i K^{\prime}-p^{\prime} K}{n} .
$$

There is no difficulty as regards the simple case of $2 n=4$.

Here

$$
\begin{aligned}
\operatorname{sn}(N u, \gamma)= & N \operatorname{sn} u\left(1-k^{2} \operatorname{sn}^{2} \frac{i K^{\prime}}{2} \operatorname{sn}^{2} u\right) \\
& \div\left(1-k^{2} \operatorname{sn}^{2} \frac{i K^{\prime}}{4} \operatorname{sn}^{2} u\right)\left(1-k^{2} \operatorname{sn}^{2} \frac{3 i K^{\prime}}{4} \sin ^{2} u\right) \\
\sin \left(N_{2} u, \gamma_{2}\right)= & N_{2} \operatorname{su} u\left(1-k^{2} \operatorname{sn}^{2} \frac{2 K-i K^{\prime}}{2} \operatorname{sn}^{2} u\right) \\
& \div\left(1-h^{2} \operatorname{sn}^{2} \frac{2 K-i K^{\prime}}{4} \sin ^{2} u\right)\left(1-k^{2} \operatorname{sn}^{2} \frac{2 K+3 i K^{\prime \prime}}{4} \sin ^{2} u\right) \\
\sin \left(N_{8} u, \gamma_{8}\right)= & N_{8} \operatorname{sn} u\left(1-k^{2} \operatorname{sn}^{2} \frac{4 K-i K^{\prime}}{2} \operatorname{sn}^{2} u\right) \\
& \div\left(1-k^{2} \sin ^{2} \frac{4 K-i K^{\prime}}{4} \sin ^{2} u\right)\left(1-k^{2} \sin ^{2} \frac{4 i+3 i K^{\prime}}{4} \sin ^{2} u\right)
\end{aligned}
$$

VOL. XVII.-NO. 254 . 
$\operatorname{sn}\left(N_{\triangleleft} u, \gamma_{s}\right)=N_{\triangleleft} \sin u\left(1-k^{2} \operatorname{sn}^{2} \frac{6 K-i K^{\prime}}{2} \operatorname{sn}^{2} u\right)$

Hence

$$
\div\left(1-k^{2} \operatorname{sn}^{2} \frac{6 K-i K^{\prime}}{4} \operatorname{sn}^{2} u\right)\left(1-k^{2} \operatorname{sn}^{2} \frac{6 K+3 i K^{\prime}}{4} \operatorname{sn}^{2} u\right)
$$

$\operatorname{am}(4 u, k)=\operatorname{am}(N u, \gamma)+\mathrm{am}\left(N_{\mathrm{g}} u, \gamma_{\mathrm{g}}\right)+\mathrm{am}\left(N_{\mathrm{s}} u, \gamma_{\mathrm{s}}\right)+\mathrm{am}\left(N_{4} u, \gamma_{\mathrm{s}}\right)$,

$\operatorname{pm}(4 u, k)=\operatorname{am}(N u, \gamma)-\operatorname{am}\left(N_{8} u, \gamma_{2}\right)+\operatorname{am}\left(N_{8} u, \gamma_{8}\right)-\operatorname{am}\left(N_{4} u, \gamma_{8}\right)$,

if

$$
\operatorname{sm}(u, k)=k \operatorname{sn}(u, k), \quad \text { am } \equiv \sin ^{-1} \mathrm{sn},
$$

and $\quad \operatorname{sn}(N u, \gamma)=N \operatorname{sn} u\left(1-k^{2} \operatorname{sn}^{2} \frac{i K^{\prime}}{2} \operatorname{sn}^{2} u\right)$

$$
\begin{array}{r}
\quad \div\left(1-k^{2} \operatorname{sn}^{2} \frac{i K^{\prime}}{4} \operatorname{sn}^{2} u\right)\left(1-k^{2} \operatorname{sn}^{2} \frac{3 i K^{\prime}}{4} \operatorname{sn}^{2} u\right) \\
=(1+\sqrt{ } k)^{3} \frac{\operatorname{sn} u(1+k \operatorname{sn} u)}{1+2 \sqrt{ } k(1+\sqrt{ } k+k) \operatorname{sn}^{2} u+k^{2} \operatorname{sn}^{6} u}
\end{array}
$$

with similar expressions for

$$
\operatorname{sn}\left(N_{2} u, \gamma_{8}\right), \quad \text { sn }\left(N_{8} u, \gamma_{8}\right) \text {, and sn }\left(N_{4} u, \gamma_{8}\right) \text {, }
$$

obtained by changing $\sqrt{ } k$ into $-i \sqrt{ } k,-\sqrt{ } k$, and $i \sqrt{ } k$, respectively.

The multiplication formula in question seems to be true when the order of the trausformations $2 n$ is of the form $2^{m}$; i:e., in that case we have

$$
\begin{gathered}
\operatorname{am}(2 u \imath, k)=\operatorname{am}(N u, \gamma)+\operatorname{ain}\left(N_{8} u, \gamma_{2}\right)+\operatorname{ain}\left(N_{8} u, \gamma_{8}\right)+\ldots \\
\ldots+\operatorname{am}\left(N_{2 n} u, \gamma_{2 n}\right) .
\end{gathered}
$$

Also, by the principle of duality, each of the above transformations gives rise to a conjugate one. With regard to these* non-real transformations, which I have considered more fully in an Appendix, it should be mentioned that if $\lambda$ and $\gamma$ be the respective moduli corres. ponding to a pair, then it is necessary to assume relations of the forms

$$
\left.\left.\begin{array}{l}
M K=2 a \Lambda+2 i b \Lambda^{\prime} \\
M K^{\prime}=a^{\prime} \Lambda^{\prime}+2 i b^{\prime} \Lambda
\end{array}\right\} \quad \begin{array}{l}
N K=a^{\prime} \Gamma+2 i b^{\prime} \Gamma^{\prime} \\
N K^{\prime}=2 a \Gamma^{\prime}+2 i b \Gamma
\end{array}\right\},
$$

where $a^{\prime}$ is an odd integer, and the other integer's are counected by

- 'This phruse is meant to include both imaginary moduli and those which are real but $>1$. 
1885:] Transformation Formula $y=\sin (L+A+B+O+\ldots) . \quad 19$

the relations $a a^{\prime}-2 b^{2}= \pm n, b+b^{\prime}=0$, if $2 n=$ order of the transformations.

[Compare this problem with Jacobi's. See Fundamenta Nova, p. 75, and a note thereon by the present writer, Proc. Lond. Math. Soc., Vol. xvi., p. 104.]

SeCr. 7.-Transformation of $\theta$ functions. Expressions for $\theta(M u, \lambda)$

$$
\text { and } \Theta(N u, \gamma) \text {. }
$$

The formula for $\theta(M u, \lambda)$, corresponding to a first real root $\lambda<1$, is $\Theta^{2 n} 0 . \Theta(M u, \lambda)=\Theta(0, \lambda) \Theta^{2 n} u \operatorname{dn} u . \Pi\left\{1-k^{2} \operatorname{sn}^{2} \frac{s K}{n} \operatorname{sn}^{3} u\right\}$

(see Proc. Lond. Math. Soc., Vol. xvi., p. 103).

From this is deduced

$$
\Theta^{2 n} 0 . \theta(N u, \gamma)=\theta(0, \gamma) \theta^{2 n} u . \Pi\left\{1-k^{2} \operatorname{sn}^{2}\left(\frac{2 t-1}{2 n} i \Pi^{\prime}\right) \operatorname{sn}^{2} u\right\} \text {, }
$$

where $s, t$ are integers from 1 to $n-1$ and 1 to $n$, as before.

This pair is, in fact, only one from a set of transformations whereof the types must be

$$
\begin{aligned}
& \theta^{2 n} 0 . \theta\left(M_{r} u, \lambda_{r}\right) \\
& =\theta\left(0, \lambda_{r}\right) \theta^{2 n} u \operatorname{dn} u . e^{-\frac{\mu_{r} u^{2}}{K^{2}}} \Pi\left\{1-k^{2} \operatorname{sn}^{2} \frac{g K+2 g^{\prime} i K^{\prime}}{n} \operatorname{sn}^{2} u\right\}
\end{aligned}
$$

( $g, g^{\prime}$ integers),

$$
\begin{aligned}
& * \Theta^{8 n} 0 . \Theta\left(N_{r} u, \gamma_{r}\right) \\
& =\theta\left(0, \gamma_{r}\right) \theta^{2 n} u . e^{-\frac{\nu_{r} t^{2}}{K^{2}}} \Pi\left\{1-k^{2} \operatorname{sn}^{2} \frac{2 m K+\left(2 m^{\prime}+1\right) i K^{\prime}}{2 n} \operatorname{sn}^{2} u\right\} .
\end{aligned}
$$

The $\mu$ and $\nu$ functions which here present themselves are of the forms

$$
\mu_{r}=\frac{\pi i b M_{r} K}{2 \Lambda_{r}}, \quad \nu_{r}=\frac{\pi i b^{\prime} N_{r} K}{2 \Gamma_{r}} .
$$

- The $\Phi$ furmule are similar to the above : e.g., in a real transformation

$$
\frac{\Phi(M u, \lambda)}{\Phi^{2 n} u} \div \frac{\Phi(0, \lambda)}{\Phi^{2 n} 0}=\frac{\Theta(M u, \lambda)}{\Theta^{2 n} u} \div \frac{\Theta(0, \lambda)}{\Theta^{2 n} 0}
$$

(For the definition of the $\Phi$-function see a note by the present writer, Proc, Royal Soc., No. 237, 1885.)

$$
\text { c } 2
$$


If we pat $u=K$, we have

$$
\gamma_{r}^{\prime}=\left(k^{\prime}\right)^{2 n} \div \Pi d n^{4} \frac{2 m K+\left(2 m^{\prime}+1\right) i K^{\prime}}{2 n} .
$$

Consequently, by the principle of duality,

$$
\lambda_{r}=k^{2 n} \div \Pi \mathrm{dn}^{4}\left(\frac{2 m K^{\prime}+\left(2 m^{\prime}+1\right) i K}{2 n}, k^{\prime}\right) .
$$

Sect. 8.-Complete multiplication by $2 n$. Expression for $\Theta 2 n u$.

It is not difficult to show that any two conjugate transformations give complete multiplication; but, as the formule are very long to write down, I here notice only a pair which corresponds to the case of $\lambda$ and $\gamma$ being each real and $<1$, viz.,

$$
\begin{gathered}
\Theta(M u, \lambda)=C \cdot \theta^{2 n} u \operatorname{dn} u . \Pi\left\{1-k^{2} \operatorname{sn}^{2} \frac{s K}{n} \operatorname{sn}^{2} u\right\}, \\
\Theta(N u, \gamma)=O . \theta^{2 n} u . \Pi\left\{1-k^{2} \operatorname{sn}^{2}\left(\frac{2 t-1}{2 n} i K^{\prime}\right) \operatorname{sn}^{2} u\right\},
\end{gathered}
$$

where $O \equiv$ a constant, generally.

Changing $\lambda, k, M$ into $k, \gamma$, and $\frac{2 n}{N}$, and $u$ into $N u$, we have from the former

$\theta 2 n u=C . \theta^{2 n}(N u, \gamma) d n(N u, \gamma) \Pi\left\{1-\gamma^{2} \operatorname{sn}^{2}\left(\frac{s \Gamma}{n}, \gamma\right) \operatorname{sn}^{2}(N u, \gamma)\right\}$, and, from Sect. 3 ,

$$
\operatorname{sn}(N u, \gamma)=N \operatorname{sn} u . P \div Q, \quad \operatorname{dn}(N u, \gamma)=R \div Q,
$$

if

$$
\left.\begin{array}{l}
\Pi\left(1+\operatorname{ct}^{2} u_{0}^{\prime} \sin ^{2} u\right)=P \\
\Pi\left(1+\operatorname{ct}^{2} v_{0}^{\prime} \operatorname{sn}^{2} u\right)=Q \\
\Pi\left(1-\operatorname{dn}^{2} v_{0}^{\prime} \operatorname{sn}^{2} u\right)=R
\end{array}\right\} .
$$

(See Notation.)

Hence

$$
\begin{aligned}
\Theta 2 n u & =O \cdot \theta^{4 n^{2}} u \cdot Q^{2 n} \cdot \frac{R}{Q} \cdot \Pi\left\{1-\gamma^{2} \operatorname{sn}^{2}\left(\frac{s \Gamma}{n}, \gamma\right) N^{2} \frac{P^{2}}{Q^{2}} \operatorname{sn}^{3} u\right\} \\
& =\text { O. } \theta^{4 n^{3}} u \cdot Q \cdot R \cdot \Pi\left\{Q^{2}-N^{2} \gamma^{2} \operatorname{sn}^{2}\left(\frac{s \Gamma}{n}, \gamma\right) P^{2} \operatorname{sn}^{2} u\right\},
\end{aligned}
$$


1885.] Transformation Formula $y=\sin (L+A+B+O+\ldots)$. 21

since $s$ is an integer from 1 to $n-1$ inclusively; or, ultimately,

$$
\Theta 2 n u=O . \theta^{4 n^{2}} u . \Pi\left\{1-a^{2} \operatorname{sn}^{2} u\right\},
$$

where the number of quadratic factors included in the symbol $I$ is

$$
2 n+(n-1) 2 n=2 n^{2} \text {. }
$$

Since the function $\theta$ vanishes for any odd multiple of $i K^{\prime}$, i.e., $\Theta(2 r+1) i K^{\prime}=0$, it is seen without difficulty that

$$
a \equiv k \operatorname{sn} \frac{2 m K+\left(2 m^{\prime}+1\right) i K^{\prime}}{2 n},
$$

if different integer values be given to $m$ and $m^{\prime}$ so as to produce the requisite $2 n^{2}$ quadratic factors. In other words, the coefficient $a$ must include all the roots of sn $2 n u=\infty$.

The above results, added to those which have been already published by the Society, give a fairly complete development of what I have called the Theory of Composition or Addition. (See Lond. Math. Soc., Vols. Xv. and Xvr.)

\section{APPENDIX.}

Remarks on the modular equation for an even transformation.

The following remarks are intended to apply more especially to those two cases where an even number is of the forms $2^{m}$ and $2 \times$ an odd prime number.

CASE $1 .-2 n=2^{m}$.

Here it is convenient to take a relation between $\lambda$ and $k$ which gives $n$ pairs of inverse moduli $\lambda, \lambda_{2}, \lambda_{3}, \lambda_{4}$, \&c., whereof one root only, viz., $\lambda$, is real and $<1$.

For example, when $2 n=2$, then

$$
\lambda=\frac{1-k^{\prime}}{1+k^{\prime \prime}} \quad \lambda_{2}=\frac{1+k^{\prime}}{1-k^{\prime}} .
$$

When $2 n=4$;

$$
\sqrt{ } \lambda=\frac{1-\sqrt{ } k^{\prime}}{1+\sqrt{ } k^{\prime \prime}} \quad \sqrt{ } \lambda_{2}=\frac{1+i \sqrt{ } k^{\prime}}{1-i \sqrt{ } k^{\prime \prime}}, \quad \sqrt{ } \lambda_{3}=\frac{1+\sqrt{ } k^{\prime}}{1-\sqrt{ } k^{\prime}},
$$

and

$$
\lambda_{4}=\frac{1-i \sqrt{ } k^{\prime}}{1+i \sqrt{ } k^{\prime}} .
$$


These correspond to

$$
\begin{gathered}
\lambda=k^{4} \div \mathrm{dn}^{4}\left(\frac{i K}{4}, k^{\prime}\right) \mathrm{dn}\left(\frac{3 i K}{4}, k^{\prime}\right), \\
\lambda_{9}=k^{4} \div \mathrm{dn}^{4}\left(\frac{2 K^{\prime}-i K}{4}, k^{\prime}\right) \mathrm{dn}\left(\frac{2 K^{\prime}+3 i K}{4}, k^{\prime}\right), \\
\lambda_{8}=k^{4} \div \mathrm{dn}^{4}\left(\frac{4 K^{\prime}-i K}{4}, k^{\prime}\right) \mathrm{dn}^{4}\left(\frac{4 K^{\prime}+3 i K}{4}, k^{\prime}\right), \\
\lambda_{4}=k^{4} \div \mathrm{dn}^{4}\left(\frac{6 K^{\prime}-i K}{4}, k^{\prime}\right) \mathrm{dn}^{4}\left(\frac{6 K^{\prime}+3 i K}{4}, k^{\prime}\right) ;
\end{gathered}
$$

where $\lambda \lambda_{3}=1, \lambda_{2} \lambda_{4}=1$.

CASE 2. - When the order of transformation $=2 \times$ an odd prime number $n$.

In this case the modular relation gives another real root, viz., $\lambda_{1}<1$, and we have accordingly what may be called the second transformation equation

$$
\begin{aligned}
& (-)^{1(n-1)} y=\sin \left\{L-A+B-\ldots+(-)^{i(n-1)}\left(A_{1}-B_{1}+\ldots\right)\right\}, \\
& \text { if } \quad a^{\prime}=\operatorname{dn} \frac{2 i K^{\prime}}{n}, \quad \beta^{\prime}=\operatorname{dn} \frac{4 i K^{\prime}}{n}, \& c . . ., \quad a^{\prime} a_{1}^{\prime}=k^{\prime}=\beta^{\prime} \beta_{1}^{\prime}=\ldots .
\end{aligned}
$$

For example, when $2 n=6$, the equation is

$$
y=\sin \left(-L+A+A_{1}\right)
$$

where

$$
a^{\prime}=\operatorname{dn} \frac{2 i K^{\prime}}{3}, \quad a_{1}^{\prime}=\operatorname{dn}\left(K-\frac{2 i K^{\prime}}{3}\right) .
$$

Corresponding to $\lambda_{1}$ we have the relations

$$
2 \Lambda_{1}=M_{1} K, \quad n \Lambda_{1}^{\prime}=M_{1} K^{\prime}
$$

Hence the principle of duality gives

$$
n \Gamma_{1}=N_{1} K \text { and } 2 \Gamma_{1}^{\prime}=N_{1} K^{\prime}
$$

(In addition to the two real roots $\lambda, \lambda_{1}$, there are $n \cdots 1$ imaginary ones.)

The following are some formulø arising from the equation, so far is I have been able to derive them, viz., 
1885.] Transformation Formula $y=\sin (L+A+B+C+\ldots)$.

$$
\begin{aligned}
& y=M_{1} x \sqrt{1-x^{3}} \Pi\left\{1-\frac{x^{2}}{\operatorname{sn}^{2} \frac{2 s i K^{\prime}}{n}}\right\}\left\{1-\frac{x^{2}}{\operatorname{sn}^{2}\left(K-\frac{2 s i K^{\prime}}{n}\right)}\right\} \div \\
& \sqrt{1-y^{8}}=\left\{1-\left(1+k^{\prime}\right) x^{2}\right\} \Pi\left\{1-\frac{x^{8}}{\operatorname{sn}^{2}\left(\frac{K}{2}+\frac{2 s i K^{\prime}}{n}\right)}\right\} \\
& \times\left\{1-\frac{x^{2}}{\operatorname{sn}^{2}\left(\frac{K}{2}-\frac{2 s i K^{\prime}}{n}\right)}\right\} \div \\
& \sqrt{1-\lambda_{1}^{2} y^{2}}=\left\{1-\left(1-k^{\prime}\right) x^{2}\right\} \Pi\left\{1-k^{2} \operatorname{sn}^{2}\left(\frac{K}{2}+\frac{2 s i K^{\prime}}{n}\right) x^{2}\right\} \\
& \times\left\{1-k^{2} \mathrm{sn}^{2}\left(\frac{K}{2}-\frac{2 s i K^{3}}{n}\right) x^{2}\right\} \div
\end{aligned}
$$

common denom. $\sqrt{1-k^{8} x^{2}} \Pi\left\{1-k^{8} \operatorname{sn}^{2} \frac{2 s i K^{\prime}}{n} x^{y}\right\}$

$$
\times\left\{1-k^{2} \operatorname{sn}^{2}\left(K-\frac{2 s i K^{\prime}}{n}\right) x^{2}\right\} ;
$$

if $y=\operatorname{sn}\left(M_{1} u, \lambda_{1}\right), x=\mathrm{sn} u$, and $s$ is now an integer from 1 to $\frac{1}{2}(n-1)$. (Order of transformation $=2 \times$ an odd prime number $n_{\text {.) }}$

$$
\begin{aligned}
& \theta^{2 n} 0 . \theta\left(M_{1} u, \lambda_{1}\right)= \theta\left(0, \lambda_{1}\right) \theta^{2 n} u \operatorname{dn} u \Pi\left\{1-k^{2} \operatorname{sn}^{2} \frac{2 s i K^{\prime}}{n} \operatorname{sn}^{2} u\right\} \\
& \times\left\{1-k^{2} \operatorname{sn}^{2}\left(K-\frac{2 s i K^{\prime}}{n}\right) \operatorname{sn}^{2} u\right\}, \\
& \theta^{2 n} 0 . \theta\left(N_{1} u, \gamma_{1}\right)= \theta\left(0, \gamma_{1}\right) \theta^{2 n} u\left(1+k \operatorname{sn}^{2} u\right) \Pi \\
& \times\left\{1-k^{2} \operatorname{sn}^{2}\left(\frac{i K^{\prime}}{2} \pm \frac{2 s K}{n}\right) \operatorname{sn}^{2} u\right\} . \\
& \lambda_{1}=k^{2 n} \div\left(1+k^{\prime}\right)^{2} \Pi \mathrm{dn}^{4}\left(\frac{i K}{2} \pm \frac{2 s K^{\prime}}{n}, k^{\prime}\right), \\
& \gamma_{1}^{\prime}=\left(k^{\prime}\right)^{2 n} \div(1+k)^{2} \Pi \mathrm{dn}^{4}\left(\frac{i K^{\prime}}{2} \pm \frac{2 s K}{n}\right), \\
&(\Pi \equiv 1, \text { when } n=1 .) \\
& N_{1}=(-)^{1(n-1)}(1+k) \Pi \mathrm{dn}^{2}\left(\frac{i K^{\prime}}{2} \pm \frac{2 s K}{n}\right) \div \Pi \mathrm{dn}^{2} \frac{2 s K}{n} \mathrm{dn}^{8}\left(i K^{\prime}+\frac{2 s K}{n}\right),
\end{aligned}
$$


24. Transformation Formula $y=\sin (L+A+B+C+\ldots)$. [Nov.12, with a corresponding expression for $M_{1}$, obtained by changing the modulus $k$ into $k^{\prime}$.

$$
\begin{aligned}
& \operatorname{sn}\left(N_{1} u, \gamma_{1}\right)=N_{1} \operatorname{sn} u \Pi\left\{1-k^{2} \operatorname{sn}^{8} \frac{2 s K}{n} \operatorname{sn}^{2} u\right\} \\
& \times\left\{1-k^{2} \operatorname{sn}^{2}\left(i K+\frac{2 s K}{n}\right) \operatorname{sn}^{2} u\right\} \\
& \div\left(1+k x^{2}\right) \Pi\left\{1-k^{2} \operatorname{sn}^{8}\left(\frac{i K^{\prime}}{2} \pm \frac{2 s K}{n}\right) \operatorname{sn}^{3} u\right\},
\end{aligned}
$$

where both the signs \pm are to be used.

As I have already observed, the non-real transformations present some difficulties; but the results of this note are, I think, in the main correct. The correlative of the transformation

$$
(-)^{1(n-1)} y=\sin \{L-A+B-\ldots\}
$$

may be studied in the form

$(-)^{i(n-1)} i z=\tan \left\{X_{0}-X_{1}+X_{2}-X_{3}+\ldots+(-)^{1(n-1)}\left(Y_{1}-Y_{9}+Y_{3}-\ldots\right)\right\}$,

where

$$
\begin{gathered}
\tan X_{0}=i \frac{(1+k) x}{1+k x^{2}}, \quad \tan X_{1}=\frac{2 i a_{1} x}{1+a_{1}^{2} x^{2}}, \ldots \ldots, \\
\tan Y_{1}=\frac{2 i b_{1} x}{1+b_{1}^{2} x^{2}}, \ldots \ldots,
\end{gathered}
$$

and the coefficients are

$$
a_{s}=\mathrm{dn} \frac{2 s K}{n} \div \operatorname{cn} \frac{2 s K}{n}, \quad b_{s}=k \operatorname{cn} \frac{2 s K}{n} \div \mathrm{dn} \frac{2 s K}{n},
$$

if $s$ be an integer from 1 to $\frac{1}{2}(n-1)$.

When $k=0$, we have

$$
(-)^{3(n-1)} i \sin n \theta=\tan \left\{X_{0}-X_{1}+X_{2}-\ldots+(-)^{i(n-1)} X_{1(n-1)}\right\} ;
$$

if $n$ be an odd prime number, and $\tan X_{0}=i \sin \theta$,

$$
\tan X_{s}=2 i \sec \frac{s \pi}{n} \sin \theta \div\left\{1+\sec ^{2} \frac{s \pi}{n} \sin ^{2} \theta\right\} \text {. }
$$

